\title{
Predictive cruise control with autonomous overtaking
}

\author{
Nikolce Murgovski and Jonas Sjöberg
}

\begin{abstract}
This paper studies the problem of optimally controlling an autonomous vehicle, to safely overtake a slowmoving leading vehicle. The problem is formulated to minimize deviation from a reference velocity and position trajectory, while keeping the vehicle on the road and avoiding collision with surrounding vehicles. We show that the optimization problem can be formulated as a convex program, by providing convex modeling steps that include change of reference frame, change of variables, sampling in relative longitudinal distance, convex relaxation and linearization. A case study is provided showing overtaking scenarios in proximity of an oncoming vehicle, and a vehicle driving on an adjacent lane and in the same direction as the leading vehicle.
\end{abstract}

\section{INTRODUCTION}

The high percentage of traffic accidents and fatalities in overtaking and lane change maneuvers, is motivating the introduction of driver assistance and fully automated maneuvering systems that are expected to increase highway safety [1]. Several of these systems, such as adaptive cruise control and lane keeping aid, are already standard in many production vehicles [2].

This paper investigates a higher level of automated driving, where the vehicle autonomously takes decisions to stay within its own lane, or to perform an overtaking maneuver in a case of a slow-moving leading vehicle. The problem can be casted as an avoidance of moving obstacles, which is typically addressed by grid/graph search techniques [3], [4], or by nonlinear, model predictive control (MPC) approaches [5], [6].

MPC approaches have shown increased attention in recent years, due to their ability to systematically handle system constraints and nonlinearities [7]. For example, a nonlinear MPC has been used for obstacle avoidance maneuvers [5], as well as for combined steering and braking control [6]. The nonlinear MPC relies on iterative solutions of optimal control problems, where the control problems are nonlinear and generally non-convex. In order to decrease computational demands of the nonlinear MPC, methods have been proposed to linearize the optimal control problem, such that a convex problem is solved in each MPC stage [8], [9]. However, it has been indicated in [8], [9] that for the studied problem, the linearization step makes the planning of the entire overtaking maneuver infeasible in a single optimization stage.

This paper studies the nonlinear and non-convex optimization problem that is to be solved at every MPC stage.

Financial support from the VINNOVA-FFI project A-drive is gratefully acknowledged.

The authors are with the Department of Signals and Systems, Chalmers University of Technology, Gothenburg, Sweden. \{nikolce.murgovski, jonas.sjoberg\}@chalmers.se.

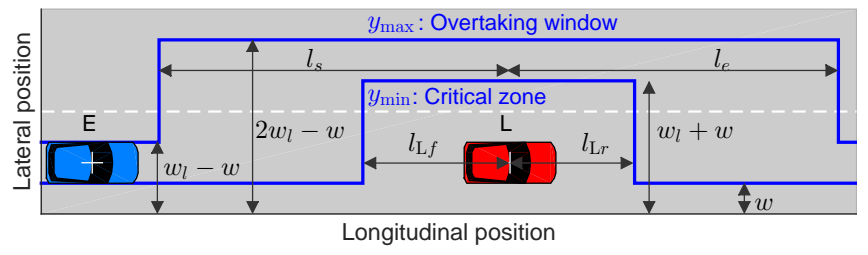

Fig. 1. Scenario where the ego vehicle (E) is overtaking a leading vehicle (L) on a road with two lanes. The center of gravity of the ego vehicle is allowed to reside between the limits $y_{\min }$ and $y_{\max }$, depicted by the thick solid lines.

The problem is first transformed into a moving reference frame and then, similarly as in [5], the time-dependent system is translated into a position-dependent system. Our contribution is showing that for a point mass vehicle model, two additional steps of convex relaxation of travel time and linearization of control input constraints yield a convex program. In comparison to previous studies, e.g. [5], [8], [9], the proposed modelling steps allow consideration of moving obstacles, such that the entire overtaking maneuver can be planned into a single optimization stage, by solving a convex quadratic program $(\mathrm{QP})$. When overtaking is performed in the neighborhood of other surrounding vehicles that drive with a different speed, or in an opposite direction of the leading vehicle, the maneuver can be planned by solving a convex second order cone program (SOCP).

The paper is organized as follows. Section II formulates the non-convex optimal control problem of overtaking a leading vehicle. In Section III modeling steps are shown that formulate the optimal control problem as a convex QP. The overtaking scenario with other surrounding vehicles is studied in Section IV. Section V provides a case study with three overtaking scenarios. The paper is ended with discussions and conclusions in Section VI.

\section{VEHICLE MODEL AND PROBLEM FORMULATION}

An overtaking scenario is studied, where an automated vehicle, which is referred to as the ego vehicle (E), is approaching a slower moving leading vehicle (L), as illustrated in Fig. 1. The ego vehicle is steered by an MPC, where the control action is re-optimized in successive stages. This paper, however, discusses in detail only a single optimization stage, with a prediction horizon of $\tilde{x}_{f}$ relative length in front of the ego vehicle. Within the prediction horizon of the stage, the road is considered straight and the leading vehicle is considered to travel with a constant longitudinal speed $v_{\mathrm{L}}$. The task of the predictive controller of the ego vehicle is to track a longitudinal reference velocity $v_{r}(t)$ and 
lateral position $y_{r}(t)$, and to safely perform an overtaking manoeuver.

1) Longitudinal and lateral dynamics: The ego vehicle is modeled as a point mass system, with state and control vectors defined as

$$
\mathbf{x}_{\mathrm{E}}(t)=\left[x_{\mathrm{E}}(t), \dot{x}_{\mathrm{E}}(t), y_{\mathrm{E}}(t)\right]^{T}, \quad \mathbf{u}_{\mathrm{E}}(t)=\left[\ddot{x}_{\mathrm{E}}(t), \dot{y}_{\mathrm{E}}(t)\right]^{T}
$$

where $x_{\mathrm{E}}$ and $y_{\mathrm{E}}$ denote longitudinal and lateral position in the inertial frame. The ego vehicle is represented by the linear system

$$
\begin{aligned}
& \dot{\mathrm{x}}_{\mathrm{E}}(t)=A \mathbf{x}_{\mathrm{E}}(t)+B \mathbf{u}_{\mathrm{E}}(t) \\
& A=\left[\begin{array}{lll}
0 & 1 & 0 \\
0 & 0 & 0 \\
0 & 0 & 0
\end{array}\right], B=\left[\begin{array}{ll}
0 & 0 \\
1 & 0 \\
0 & 1
\end{array}\right] .
\end{aligned}
$$

2) State and control constraints: Constraints are imposed on the state and control signals of the ego vehicle

$$
\mathbf{x}_{\mathrm{E}}(t) \in\left[\mathbf{x}_{\min }(\cdot), \mathbf{x}_{\max }(\cdot)\right], \quad \mathbf{u}_{\mathrm{E}}(t) \in\left[\mathbf{u}_{\min }(t), \mathbf{u}_{\max }(t)\right],
$$

with the limits defined as

$$
\begin{aligned}
& \mathbf{x}_{\min }(\cdot)=\left[0, v_{\mathrm{L}}+\epsilon, y_{\min }(\cdot)\right]^{T} \\
& \mathbf{x}_{\max }(\cdot)=\left[\text { free, } v_{x \max }(t), y_{\max }(\cdot)\right]^{T} \\
& \mathbf{u}_{\min }(t)=\left[a_{x \min }(t), v_{y \min }(t)\right]^{T} \\
& \mathbf{u}_{\max }(t)=\left[a_{x \max }(t), v_{y \max }(t)\right]^{T}
\end{aligned}
$$

where we use $(\cdot)$ as a shorthand notation for a function of decision variables. Here, $\epsilon$ is a small positive number that indicates a strict inequality, i.e., $\dot{x}_{\mathrm{E}}(t)>v_{\mathrm{L}} \geq 0$, $\forall t \in\left[0, t_{f}\right]$, which clearly states that the scenario of interest is when the leading vehicle is slower than the ego vehicle. The final time $t_{f}$ and the final position $x_{f}$ are free, while the vehicle is initially placed at zero time and zero position. The speed and acceleration limits satisfy $v_{y \min }(t), a_{x \min }(t) \leq 0$, $v_{y \max }(t), v_{x \max }(t), a_{x \max }(t) \geq 0, \forall t \in\left[0, t_{f}\right]$.

The lateral limits

$$
\begin{aligned}
& y_{\min }(\cdot)= \begin{cases}w_{l}+w, & x_{\mathrm{E}}(t) \in x_{\mathrm{L}}(t)+\left[-l_{\mathrm{L} f}, l_{\mathrm{L} r}\right] \\
w, & \text { otherwise }\end{cases} \\
& y_{\max }(\cdot)= \begin{cases}2 w_{l}-w, & x_{\mathrm{E}}(t) \in x_{\mathrm{L}}(t)+\left[-l_{s}, l_{e}\right] \\
w_{l}-w, & \text { otherwise }\end{cases}
\end{aligned}
$$

are enforced to keep the ego vehicle on the road and provide safe overtaking. The position of the leading vehicle is computed as $x_{\mathrm{L}}(t)=x_{\mathrm{L} 0}+v_{\mathrm{L}} t$, where $x_{\mathrm{L} 0}$ is its initial position. The center of gravity of the ego vehicle is prevented to enter the critical zone $\left[-l_{\mathrm{L} f}, l_{\mathrm{L} r}\right]$ around the leading vehicle. The zone is here represented as a rectangle, although for the method being discussed the zone may have any other shape. The coefficients $w_{l}$ and $w$ represent lane width and lateral safety margin, respectively. The remaining coefficients $l_{\mathrm{L} f}$, $l_{\mathrm{L} r}, l_{s}, l_{e}$ denote longitudinal lengths. They are computed as a function of the difference between the mean reference speed of the ego vehicle and the speed of the leading vehicle, and are constant within the optimization stage. The latter two lengths are introduced to permit overtaking only within a certain window $\left[-l_{s}, l_{e}\right]$ around the leading vehicle.
Additionally, a constraint is imposed on lateral speed,

$$
\dot{y}_{\mathrm{E}}(t) \in\left[s_{\min }, s_{\max }\right] \dot{x}_{\mathrm{E}}(t)
$$

which requires longitudinal motion in order to perform any change in lateral movement. Here, $s_{\min }=-\arctan \beta$, $s_{\text {max }}=\arctan \beta$, are functions of a slip angle $\beta$, where the slip angle is chosen to ensure generation of a path that can be followed by a car in a cartesian coordinate system [8].

3) Problem statement: We can now formulate the optimization problem as

$$
\begin{aligned}
& \min _{\mathbf{u}_{\mathrm{E}}(t)} J\left(\mathbf{x}_{\mathrm{E}}(t), \mathbf{u}_{\mathrm{E}}(t), \dot{\mathbf{u}}_{\mathrm{E}}(t)\right) \\
& \text { subject to } \\
& \dot{\mathbf{x}}_{\mathrm{E}}(t)=A \mathbf{x}_{\mathrm{E}}(t)+B \mathbf{u}_{\mathrm{E}}(t) \\
& \mathbf{x}_{\mathrm{E}}(t) \in\left[\mathbf{x}_{\min }(\cdot), \mathbf{x}_{\max }(\cdot)\right] \\
& \mathbf{u}_{\mathrm{E}}(t) \in\left[\mathbf{u}_{\min }(t), \mathbf{u}_{\max }(t)\right] \\
& \mathbf{x}_{\mathrm{E}}(0)=\mathbf{x}_{\mathrm{E} \mathbf{0}} \\
& \dot{y}_{\mathrm{E}}(t) \in\left[s_{\min }, s_{\max }\right] \dot{x}_{\mathrm{E}}(t)
\end{aligned}
$$

where the constraints are imposed elementwise, $\forall t \in\left[0, t_{f}\right]$. The initial state values are denoted by $\mathbf{x}_{\mathrm{E} 0}=\left[x_{\mathrm{E} 0}, v_{\mathrm{E} 0}, y_{\mathrm{E} 0}\right]^{T}$ and satisfy $\mathbf{x}_{\mathrm{E} \mathbf{0}} \in\left[\mathbf{x}_{\min }(t=0), \mathbf{x}_{\max }(t=0)\right]$. The objective $J(\cdot)$ includes penalties for deviation from the reference velocity $v_{r}(t)$ and reference position $y_{r}(t)$, and may also include cost for longitudinal acceleration and lateral speed, cost for final state values, cost for the final time, etc. Detailed implementation of this function is deferred to Section III-D. Optimization variables are the control trajectories $\mathbf{u}_{\mathrm{E}}(t)$.

The optimization problem (8) is non-convex and mixedinteger. Non-convexity arises in the state limits (8c), i.e. in the mixed-integer lateral limits (6). In Section III, we provide modeling steps for an exact convex reformulation of (6).

\section{CONVEX MODELING}

The problem (8) is reformulated here as a convex program. Convex modeling steps are performed, including change of reference frame, change of variables, sampling in relative distance and linearization.

\section{A. Change of reference frame}

The problem (8) is transformed here into a reference frame that moves longitudinally with speed $-v_{\mathrm{L}}$ relative to the road frame. This transformation allows the system to be viewed as a projection on a TV screen, where the transformed reference frame represents the TV frame in which the leading vehicle appears stationary, while the road moves towards the ego vehicle with speed $-v_{\mathrm{L}}$. We consider the origin of the moving frame located at the initial position of the ego vehicle, at time $t=0$. Thus, the position of the ego vehicle in the moving frame can be expressed by subtracting the vector $\mathbf{p}_{\mathrm{L}}(t)=\left[v_{\mathrm{L}} t, v_{\mathrm{L}}, 0\right]^{T}$ from the vehicle's state vector. The transformation is implemented as a variable change

$$
\tilde{\mathbf{x}}_{\mathrm{E}}(t)=\mathbf{x}_{\mathrm{E}}(t)-\mathbf{p}_{\mathrm{L}}(t), \quad \tilde{\mathbf{u}}_{\mathrm{E}}(t)=\left[\ddot{\tilde{x}}_{\mathrm{E}}(t), \dot{y}_{\mathrm{E}}(t)\right]^{T}
$$


where $\tilde{x}_{\mathrm{E}}(t)=x_{\mathrm{E}}(t)-v_{\mathrm{L}} t$ is the longitudinal position of the ego vehicle relative to the leading vehicle.

With the use of the latest variable change, the original problem formulation (8) is translated to a formulation in the moving reference frame

$$
\begin{aligned}
& \min _{\tilde{\mathbf{u}}_{\mathrm{E}}(t)} J\left(\tilde{\mathbf{x}}_{\mathrm{E}}(t), \tilde{\mathbf{u}}_{\mathrm{E}}(t), \dot{\tilde{\mathbf{u}}}_{\mathrm{E}}(t)\right) \\
& \text { subject to } \\
& \dot{\tilde{\mathbf{x}}}_{\mathrm{E}}(t)=A \tilde{\mathbf{x}}_{\mathrm{E}}(t)+B \tilde{\mathbf{u}}_{\mathrm{E}}(t) \\
& \tilde{\mathbf{x}}_{\mathrm{E}}(t) \in\left[\mathbf{x}_{\min }(\cdot), \mathbf{x}_{\max }(\cdot)\right]-\mathbf{p}_{\mathrm{L}}(t) \\
& \tilde{\mathbf{u}}_{\mathrm{E}}(t) \in\left[\mathbf{u}_{\min }(t), \mathbf{u}_{\max }(t)\right] \\
& \tilde{\mathbf{x}}_{\mathrm{E}}(0)=\mathbf{x}_{\mathrm{E} \mathbf{0}}-\mathbf{p}_{\mathrm{L}}(0) \\
& \dot{y}_{\mathrm{E}}(t) \in\left[s_{\min }, s_{\max }\right]\left(\dot{\tilde{x}}_{\mathrm{E}}(t)+v_{\mathrm{L}}\right)
\end{aligned}
$$

where the matrices $A$ and $B$ are exactly as in (1).

The lateral limits in (10c) are now expressed as

$$
\begin{aligned}
& y_{\min }(\cdot)= \begin{cases}w_{l}+w, & \tilde{x}_{\mathrm{E}}(t) \in x_{\mathrm{L} 0}+\left[-l_{\mathrm{L} f}, l_{\mathrm{L} r}\right] \\
w, & \text { otherwise }\end{cases} \\
& y_{\max }(\cdot)= \begin{cases}2 w_{l}-w, & \tilde{x}_{\mathrm{E}}(t) \in x_{\mathrm{L} 0}+\left[-l_{s}, l_{e}\right] \\
w_{l}-w, & \text { otherwise. }\end{cases}
\end{aligned}
$$

With this transformation, the rectangular regions around the leading vehicle appear stationary in the moving frame. However, the set (10c) is still not convex, since the time the ego vehicle will approach the critical zone depends on the optimal speed of the ego vehicle that is yet to be determined. A reformulation that will finally transform (10c) to a convex constraint is provided in Section III-B.

\section{B. Sampling in relative longitudinal distance}

The optimization problem is reformulated here into a spatial domain, by sampling into the longitudinal distance, $\tilde{x}$, in the moving frame. The shorthand notation $(\cdot)^{\prime}$ is used to denote a derivative with respect to distance, i.e., $y^{\prime}=d y / d \tilde{x}$.

An immediate consequence from the transformation in spatial domain is that the position of the ego vehicle can be removed from the state vector. The state and control vectors are now defined as

$$
\hat{\mathbf{x}}_{\mathrm{E}}(\tilde{x})=\left[\dot{\tilde{x}}_{\mathrm{E}}(\tilde{x}), y_{\mathrm{E}}(\tilde{x})\right]^{T}, \quad \hat{\mathbf{u}}_{\mathrm{E}}(\tilde{x})=\left[\dot{\tilde{x}}_{\mathrm{E}}^{\prime}(\tilde{x}), y_{\mathrm{E}}^{\prime}(\tilde{x})\right]^{T}
$$

and the ego vehicle is presented by the simple linear system $\hat{\mathbf{x}}_{\mathrm{E}}^{\prime}(\tilde{x})=\hat{\mathbf{u}}_{\mathrm{E}}(\tilde{x})$. The lateral limits (11) are now completely defined as functions of the spatial coordinate $\tilde{x}$, i.e.,

$$
\begin{aligned}
& y_{\min }(\tilde{x})= \begin{cases}w_{l}+w, & \tilde{x} \in x_{\mathrm{L} 0}+\left[-l_{\mathrm{L} f}, l_{\mathrm{L} r}\right] \\
w, & \text { otherwise }\end{cases} \\
& y_{\max }(\tilde{x})= \begin{cases}2 w_{l}-w, & \tilde{x} \in x_{\mathrm{L} 0}+\left[-l_{s}, l_{e}\right] \\
w_{l}-w, & \text { otherwise }\end{cases}
\end{aligned}
$$

Therefore, the state constraints $\hat{\mathbf{x}}_{\mathrm{E}}(\tilde{x}) \in\left[\hat{\mathbf{x}}_{\min }(\tilde{x}), \hat{\mathbf{x}}_{\max }(\tilde{x})\right]$, with the limits defined as

$$
\begin{aligned}
& \hat{\mathbf{x}}_{\min }(\tilde{x})=\left[\epsilon, y_{\min }(\tilde{x})\right]^{T} \\
& \hat{\mathbf{x}}_{\max }(\tilde{x})=\left[v_{x \max }(\tilde{x})-v_{\mathrm{L}}, y_{\max }(\tilde{x})\right]^{T}
\end{aligned}
$$

become convex, linear constraints. Non-convexity is introduced at the control constraints (10d) and the lateral slip constraint (10f), which in the spatial domain translate to

$$
\begin{aligned}
& \dot{\tilde{x}}_{\mathrm{E}}^{\prime}(\tilde{x}) \in\left[a_{x \min }(\tilde{x}), a_{x \max }(\tilde{x})\right] / \dot{\tilde{x}}_{\mathrm{E}}(\tilde{x}) \\
& y_{\mathrm{E}}^{\prime}(\tilde{x}) \in\left[v_{y \min }(\tilde{x}), v_{y \max }(\tilde{x})\right] / \dot{\tilde{x}}_{\mathrm{E}}(\tilde{x}) \\
& y_{\mathrm{E}}^{\prime}(\tilde{x}) \in\left[s_{\min }, s_{\max }\right]\left(1+v_{\mathrm{L}} / \dot{\tilde{x}}_{\mathrm{E}}(\tilde{x})\right) .
\end{aligned}
$$

This, however, can be easily handled by the standard MPC technique of linearization, as it will be shown in Section III-C.

\section{Linearization and convex problem formulation}

The constraints (15)-(17) can be modeled as convex by linearizing the term $1 / \dot{\tilde{x}}_{\mathrm{E}}(\tilde{x})$ about the relative reference velocity $\tilde{v}_{r}(\tilde{x})=v_{r}(\tilde{x})-v_{\mathrm{L}}$. By using

$$
1 / \dot{\tilde{x}}_{\mathrm{E}}(\tilde{x}) \approx \frac{1}{\tilde{v}_{r}(\tilde{x})}\left(2-\frac{\dot{\tilde{x}}_{\mathrm{E}}(\tilde{x})}{\tilde{v}_{r}(\tilde{x})}\right)
$$

the optimization problem can be formulated with linear constraints,

$$
\begin{aligned}
& \min _{\hat{\mathbf{u}}_{\mathrm{E}}(\tilde{x})} J\left(\hat{\mathbf{x}}_{\mathrm{E}}(\tilde{x}), \hat{\mathbf{u}}_{\mathrm{E}}(\tilde{x}), \hat{\mathbf{u}}_{\mathrm{E}}^{\prime}(t)\right) \\
& \text { subject to } \\
& \hat{\mathbf{x}}_{\mathrm{E}}^{\prime}(\tilde{x})=\hat{\mathbf{u}}_{\mathrm{E}}(\tilde{x}) \\
& \hat{\mathbf{x}}_{\mathrm{E}}(\tilde{x}) \in\left[\hat{\mathbf{x}}_{\min }(\tilde{x}), \hat{\mathbf{x}}_{\max }(\tilde{x})\right] \\
& \hat{\mathbf{u}}_{\mathrm{E}}(\tilde{x}) \in\left[\mathbf{u}_{\min }(\tilde{x}), \mathbf{u}_{\max }(\tilde{x})\right] \frac{1}{\tilde{v}_{r}(\tilde{x})}\left(2-\frac{\dot{\tilde{x}}_{\mathrm{E}}(\tilde{x})}{\tilde{v}_{r}(\tilde{x})}\right) \\
& \hat{\mathbf{x}}_{\mathrm{E}}(0)=\hat{\mathbf{x}}_{\mathrm{E} \mathbf{0}} \\
& y_{\mathrm{E}}^{\prime}(\tilde{x}) \in\left[s_{\min }, s_{\max }\right]\left(1+\frac{v_{\mathrm{L}}}{\tilde{v}_{r}(\tilde{x})}\left(2-\frac{\dot{\tilde{x}}_{\mathrm{E}}(\tilde{x})}{\tilde{v}_{r}(\tilde{x})}\right)\right)
\end{aligned}
$$

where the constraints are enforced for all $\tilde{x} \in\left[0, \tilde{x}_{f}\right]$, and the initial state vector is defined as $\hat{\mathbf{x}}_{\mathrm{E} 0}=\left[v_{\mathrm{E} 0}-v_{\mathrm{L}}, y_{\mathrm{E} 0}\right]^{T}$. The linearized input constraints (19d), (19f) are an inner approximation of the feasible set, thus ensuring a feasible control trajectory in the original formulation (8). If any of these constraints is activated along the optimization horizon, then the obtained solution is conservative and may not be optimal to the original formulation. The optimization error can be decreased by re-linearizing about the optimal velocity trajectory, when the control problem is to be solved iteratively. This is a standard MPC practice [7].

Finally, if the cost function $J(\cdot)$ is chosen to be convex, the optimization problem (19) would be a convex program.

\section{Convex objective function}

The performance of the system is evaluated by a quadratic cost function

$$
\begin{aligned}
J(\cdot) & =\int_{0}^{\tilde{x}_{f}}\left\|\hat{\mathbf{x}}_{\mathrm{E}}(\tilde{x})-\hat{\mathbf{x}}_{\mathbf{r}}(\tilde{x})\right\|_{Q}^{2} d \tilde{x} \\
& +\int_{0}^{\tilde{x}_{f}}\left(\left\|\hat{\mathbf{u}}_{\mathrm{E}}(\tilde{x})\right\|_{R}^{2}+\left\|\hat{\mathbf{u}}_{\mathrm{E}}^{\prime}(\tilde{x})\right\|_{S}^{2}\right) d \tilde{x}
\end{aligned}
$$

where $\hat{\mathbf{x}}_{\mathbf{r}}(\tilde{x})=\left[\tilde{v}_{r}(\tilde{x}), y_{r}(\tilde{x})\right]^{T}$ is the vector of reference trajectories, and $Q \geq 0, R>0, S \geq 0$ are block diagonal 


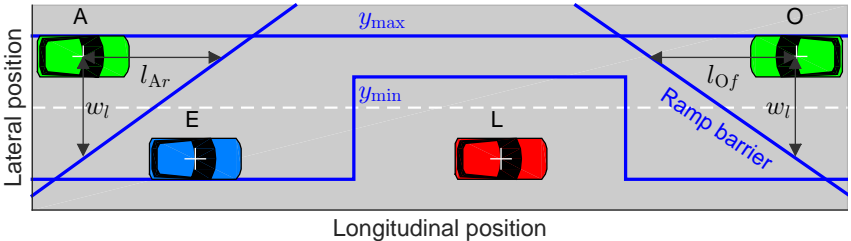

Fig. 2. Overtaking scenarios with an oncoming vehicle (O) and with an adjacent vehicle (A) moving in the same direction on an adjacent lane. During the overtaking maneuver, the center of gravity of the ego vehicle is allowed to reside among the limits $y_{\min }, y_{\max }$ and the ramp barrier of the oncoming or adjacent vehicle.

weighting matrices of appropriate dimensions, penalizing deviations from the reference trajectories, the control actions and changes in the control actions.

\section{SCENARIOS WITH SURROUNDING VEHICLES}

Realistic overtaking scenarios may involve more than one vehicle in the neighborhood of the ego vehicle. If these vehicles move in the same direction and with the same speed as the leading vehicle, then in the moving reference frame each vehicle will create a stationary critical zone that can be included in the computation of the lateral limits (12), without infringing the convexity of problem (19). Here, we investigate other two scenarios, where a surrounding vehicle drives with a different speed, or in an opposite direction of the leading vehicle.

\section{A. Overtaking in proximity of an oncoming vehicle}

Consider an oncoming vehicle $(\mathrm{O})$, driving with a constant speed $v_{\mathrm{O}} \leq 0$ and at a constant lateral position $y_{\mathrm{O}}=3 w_{l} / 2$. Negative speed indicates a motion in the opposite direction of the leading vehicle, as illustrated in Fig. 2. Using a rectangular critical zone around the oncoming vehicle will infringe problem convexity, since the critical zone will not appear stationary in the moving frame. To remedy the issue, we apply a convexification step, previously proposed by [8] The strategy is to model the critical zone of the oncoming vehicle as a ramp barrier, as illustrated in Fig. 2.

1) Travel time as a state: To model the ramp barrier constraint, we introduce the travel time $\tilde{t}(\tilde{x})$ in the moving frame as an additional state in the problem, for which it holds $\tilde{t}^{\prime}(\tilde{x})=1 / \dot{\tilde{x}}_{\mathrm{E}}(\tilde{x})$. Note that, this is not a linear function and an equality constraint of this form is not convex. The constraint could be convexified in several ways, but besides standard linearization techniques, we propose here a convex relaxation

$$
\tilde{t}^{\prime}(\tilde{x}) \geq 1 / \dot{\tilde{x}}_{\mathrm{E}}(\tilde{x}), \forall \tilde{x} \in\left[0, \tilde{x}_{f}\right],
$$

the validation of which is deferred to Section IV-A.4, after the entire problem of overtaking with an oncoming vehicle is being formulated.

2) Ramp barrier constraint: The ramp barrier of the oncoming vehicle can now be formulated as a simple linear constraint

$$
\frac{\tilde{x}-x_{\mathrm{O} 0}-\left(v_{\mathrm{O}}-v_{\mathrm{L}}\right) \tilde{t}(\tilde{x})}{l_{\mathrm{O} f}}+\frac{y_{\mathrm{E}}(\tilde{x})-y_{\mathrm{O}}}{w_{l}} \leq-1
$$

where $x_{\mathrm{O} 0}$ is the initial position of the oncoming vehicle and $l_{\mathrm{O} f}$, which is constant in the optimization stage, is a longitudinal length computed as a function of the difference between the mean reference speed of the ego vehicle and the speed of the oncoming vehicle. The constraint (22) is clearly convex, but if imposed at each sampling instance, it will forbid the entire half plane, northeast of the oncoming vehicle, thus not allowing the planning of the entire overtaking maneuver in a single optimization stage, as originally indicated in [8]. While this has been a serious burden in [8], [9], here it can be easily handled by simply switching off the constraint (22) when the ego vehicle is outside the overtaking window. Namely, outside the overtaking window, the ego vehicle is constrained by $y_{\max }(\tilde{x})$ to stay within its own lane (see Fig. 1), where the oncoming vehicle is not a hazard to the ego vehicle.

3) Problem statement: Finally, the convex optimization problem of overtaking with an oncoming vehicle can be formulated as

$$
\min _{\hat{\mathbf{u}}_{\mathrm{E}}(\tilde{x})} J\left(\hat{\mathbf{x}}_{\mathrm{E}}(\tilde{x}), \hat{\mathbf{u}}_{\mathrm{E}}(\tilde{x}), \hat{\mathbf{u}}_{\mathrm{E}}^{\prime}(t)\right)+\epsilon \tilde{t}\left(\tilde{x}_{f}\right)
$$

subject to

$$
\begin{aligned}
& \tilde{t}^{\prime}(\tilde{x}) \geq 1 / \dot{\tilde{x}}_{\mathrm{E}}(\tilde{x}) \\
& \hat{\mathbf{x}}_{\mathrm{E}}^{\prime}(\tilde{x})=\hat{\mathbf{u}}_{\mathrm{E}}(\tilde{x}) \\
& \hat{\mathbf{x}}_{\mathrm{E}}(\tilde{x}) \in\left[\hat{\mathbf{x}}_{\min }(\tilde{x}), \hat{\mathbf{x}}_{\max }(\tilde{x})\right] \\
& \hat{\mathbf{u}}_{\mathrm{E}}(\tilde{x}) \in\left[\mathbf{u}_{\min }(\tilde{x}), \mathbf{u}_{\max }(\tilde{x})\right] \frac{1}{\tilde{v}_{r}(\tilde{x})}\left(2-\frac{\dot{\tilde{x}}_{\mathrm{E}}(\tilde{x})}{\tilde{v}_{r}(\tilde{x})}\right) \\
& \hat{\mathbf{x}}_{\mathrm{E}}(0)=\hat{\mathbf{x}}_{\mathrm{E} \mathbf{0}} \\
& y_{\mathrm{E}}^{\prime}(\tilde{x}) \in\left[s_{\min }, s_{\max }\right]\left(1+\frac{v_{\mathrm{L}}}{\tilde{v}_{r}(\tilde{x})}\left(2-\frac{\dot{\tilde{x}}_{\mathrm{E}}(\tilde{x})}{\tilde{v}_{r}(\tilde{x})}\right)\right) \\
& \frac{\tilde{x}-x_{\mathrm{O} 0}-\left(v_{\mathrm{O}}-v_{\mathrm{L}}\right) \tilde{t}(\tilde{x})}{l_{\mathrm{O} f}}+\frac{y_{\mathrm{E}}(\tilde{x})-y_{\mathrm{O}}}{w_{l}} \leq-1
\end{aligned}
$$

where constraints (23b)-(23g) are imposed for all $\tilde{x} \in\left[0, \tilde{x}_{f}\right]$, while (23h) is imposed for $\tilde{x} \in x_{\mathrm{L} 0}+\left[-l_{s}, l_{e}\right]$. Note that, the objective function has been augmented with the term $\epsilon \tilde{t}\left(\tilde{x}_{f}\right)$, where $\epsilon$ is a small positive number. The reason for introducing this penalty is to give an incentive for obtaining tight equality of (23b) at the optimum, even when the ramp barrier constraint (23h) is not activated. Also note that, penalizing travel time, which is not trivial in the timedependent formulation, is straightforward to introduce in the spatial formulation.

The problem (23) is not a QP, even when the objective function is quadratic. The problem is a SOCP, since the constraint (23b) can be represented as a second order cone constraint [10]. A QP formulation can be obtained by linearizing (23b) about the reference velocity $v_{r}(\tilde{x})$. This, however, may introduce noteworthy approximation error, since (23b) will be active at each sampling instance within the overtaking window. The error can be kept within certain bounds by frequently re-optimizing and re-linearizing in successive MPC stages.

4) Validity of the travel time relaxation: 
Proposition. The global minimum of the non-convex version of problem (23), where (23b) is not relaxed, can be obtained by solving the relaxed convex problem (23).

Proof. We will prove the proposition by contradiction. Suppose that an optimal solution of problem (23) is found for which it holds $\tilde{t}^{\prime *}(\tilde{x})=1 / \dot{\tilde{x}}_{\mathrm{E}}^{*}(\tilde{x})+\delta(\tilde{x})$, where $\delta(\tilde{x})$ is a slack variable that is nonnegative for all $\tilde{x} \in\left[0, \tilde{x}_{f}\right]$. Then, it is possible to construct an alternative suboptimal solution with the same optimal control signals $\hat{\mathbf{u}}_{\mathrm{E}}^{*}(\tilde{x})$, but with a state derivative trajectory $\check{t}^{\prime}(\tilde{x})=\tilde{t}^{\prime *}(\tilde{x})-\delta(\tilde{x})$. Defining such a trajectory is a feasible solution, indeed, since the ramp barrier constraint

$$
\frac{\tilde{x}-x_{\mathrm{O} 0}-\left(v_{\mathrm{O}}-v_{\mathrm{L}}\right) \check{t}^{\prime}(\tilde{x})}{l_{\mathrm{O} f}}+\frac{y_{\mathrm{E}}(\tilde{x})-y_{\mathrm{O}}}{w_{l}} \leq-1
$$

will not be violated, because the vector on the left side of the inequality is less than or equal (for all $\tilde{x} \in\left[0, \tilde{x}_{f}\right]$ ) to the vector of the feasible (and optimal) solution where $\tilde{t}^{\prime *}(\tilde{x})$ is used. However, the final time of this feasible solution satisfies $\check{t}\left(\tilde{x}_{f}\right)=\tilde{t}^{*}\left(\tilde{x}_{f}\right)-\int_{0}^{\tilde{x}_{f}} \delta(\tilde{x}) d \tilde{x} \leq \tilde{t}^{*}\left(\tilde{x}_{f}\right)$, which directly implies from the objective (23a) that the solution with nonzero slack variables, $\tilde{t}^{\prime *}(\tilde{x})$, could not have been optimal, but rather the solution where the slack variables are zero is the optimal.

\section{B. Overtaking in proximity of a vehicle on an adjacent lane}

Consider an overtaking scenario with a surrounding vehicle (A) driving with a constant speed $v_{\mathrm{A}}>v_{\mathrm{L}}$ on an adjacent lane and with the same direction as the ego vehicle. The adjacent vehicle lateral position is $y_{\mathrm{A}}=3 w_{l} / 2$, while its initial longitudinal position $x_{\mathrm{A} 0}$ is outside the overtaking window, such that the ego vehicle may realize the overtaking maneuver before the adjacent vehicle passes by the leading vehicle. Similarly as with the oncoming vehicle, the constraint on critical zone is modeled by a ramp barrier

$$
\frac{\tilde{x}-x_{\mathrm{A} 0}-\left(v_{\mathrm{A}}-v_{\mathrm{L}}\right) \tilde{t}(\tilde{x})}{l_{\mathrm{A} r}}-\frac{y_{\mathrm{E}}(\tilde{x})-y_{\mathrm{A}}}{w_{l}} \geq 1
$$

which is imposed for $\tilde{x} \in x_{\mathrm{L} 0}+\left[-l_{s}, l_{e}\right]$. The length $l_{\mathrm{A} r}$ is computed as a function of the difference between the mean reference speed of the ego vehicle and the speed of the adjacent vehicle and is constant within the optimization stage.

The problem formulation for this scenario is exactly as problem (23), but with the constraint (23h) replaced by (25).

\section{CASE STUdy: OVERTAKING IN THREE SCENARIOS}

We provide here a case study of three overtaking scenarios. In the first scenario, the ego vehicle, with a reference speed of $70 \mathrm{~km} / \mathrm{h}$, is overtaking a leading vehicle, with speed of $50 \mathrm{~km} / \mathrm{h}$, without the presence of other surrounding vehicles. In the second scenario, the overtaking takes place in the proximity of an oncoming vehicle, which is driving with $70 \mathrm{~km} / \mathrm{h}$ in the opposite direction of the leading vehicle. In the third scenario, the ego vehicle is about to overtake the leading vehicle, before a vehicle driving with $70 \mathrm{~km} / \mathrm{h}$ on an adjacent lane and in the same direction as the ego vehicle,
TABLE I

PROBLEM DATA.
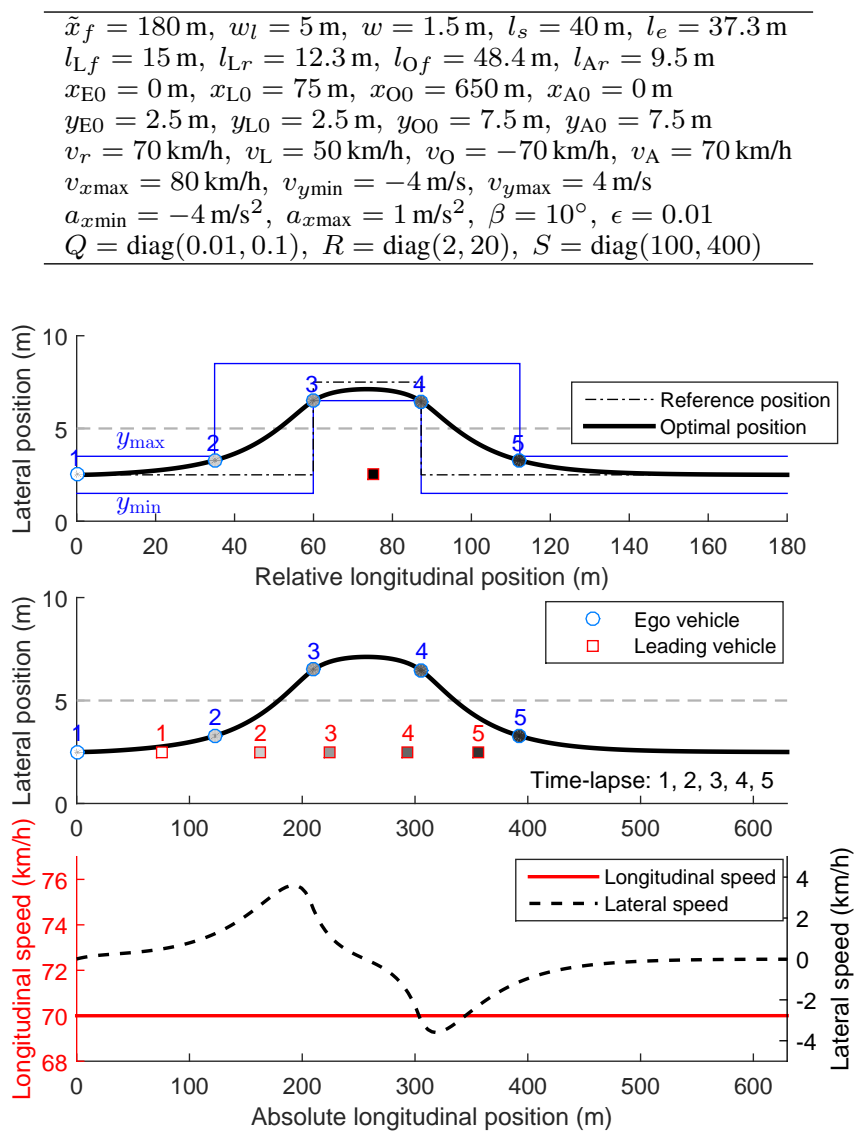

Fig. 3. Overtaking scenario where the ego vehicle, driving with $70 \mathrm{~km} / \mathrm{h}$, is overtaking a leading vehicle, driving with $50 \mathrm{~km} / \mathrm{h}$. The top plot shows the relative position of the ego vehicle in the moving frame, while the other two plots show the absolute position and velocity in the inertial frame. A timelapse is shown with five time instances, 1) the initial time, 2) the time when the ego vehicle is positioned at the beginning of the overtaking window, 3) the beginning of the critical zone of the leading vehicle, 4) the end of the critical zone, and 5) the end of the overtaking window.

passes by the leading vehicle. The remaining problem data is provided in Table I.

The optimal control problem (23) is transferred to a discrete form using first order Euler discretization, with sampling interval of $1 \mathrm{~m}$ relative distance. The problem is then automatically translated to a standard SOCP, by using the CVX modeling language [11], [12]. The problem is solved with the SOCP solver ECOS [13], for which CVX reported an average computation time of about $70 \mathrm{~ms}$ spent on the solver, on a PC with $2.67 \mathrm{GHz}$ dual-core processor and 4 GB RAM. Note, however, that the computation time can be decreased by linearizing (23b) and solving the problem with a dedicated QP solver, instead of the generalized SOCP solver.

In all three scenarios, the reference lateral position of the ego vehicle is assigned at the middle of its own lane, except within the critical zone of the leading vehicle, where the reference position is at the middle of the adjacent lane, as depicted in Fig. 3. It can be observed in Fig. $3-5$, that the 

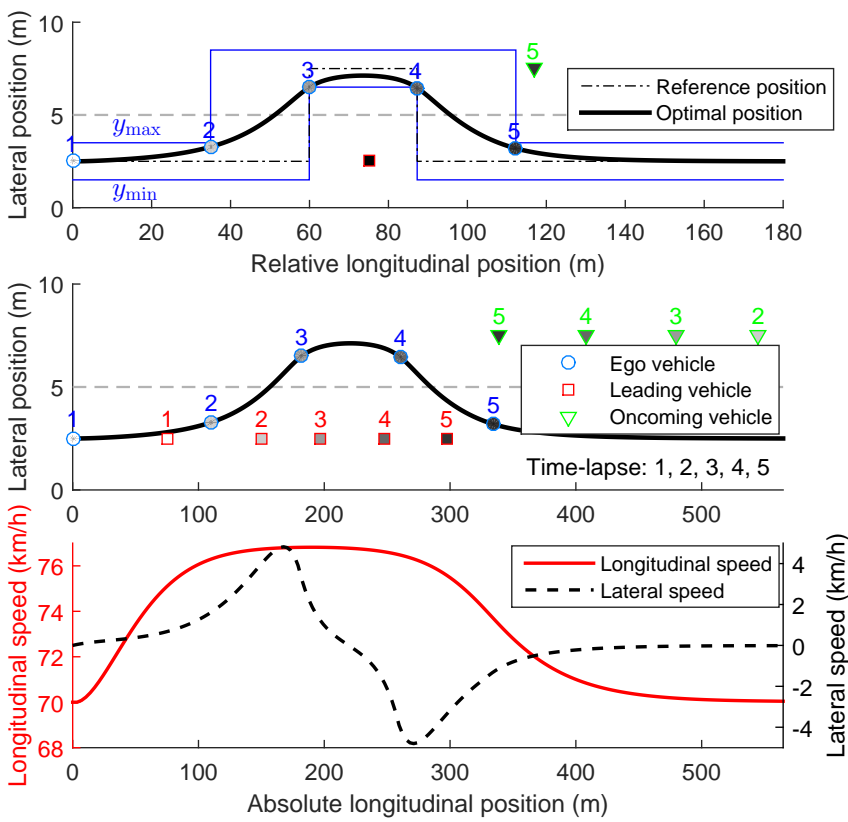

Fig. 4. Overtaking scenario in the proximity of an oncoming vehicle driving with $70 \mathrm{~km} / \mathrm{h}$. The ego vehicle accelerates to about $76.5 \mathrm{~km} / \mathrm{h}$ in order to overtake the leading vehicle and return to its own lane, before activating the ramp barrier of the oncoming vehicle.

optimal position trajectory of the ego vehicle is smooth in all three scenarios. The longitudinal velocity of the ego vehicle in the first scenario is equal to the reference, while in the remaining two scenarios the ego vehicle accelerates in order to avoid a collision with the oncoming or adjacent vehicle.

The figures also show a time-lapse with five time instances, where the marker depicting each vehicle is shaded from a lighter to a darker color as the time elapses. It can be observed in the top plots that in the moving frame, the leading vehicles appears stationery as time passes, while the remaining vehicles move with a relative speed with respect to the leading vehicle.

\section{DISCUSSION AND CONCLUSION}

This paper provides convex modeling steps for the problem of optimal overtaking within the scope of a predictive cruise control. We show that the originally non-convex optimal control problem, can be formulated as a convex program that can be solved efficiently. The resulting convex program is a $\mathrm{QP}$, when all surrounding vehicles drive with the same speed as the leading vehicle. When a surrounding vehicle drives with a different speed, or in an opposite direction of the leading vehicle, the convex program is a SOCP, which could be approximated to a QP by linearizing one constraint.

Future studies may focus on extending the proposed approach on overtaking scenarios with curvy roads, with surrounding vehicles that may not drive with constant speed, and with measurements that may include sensor noise and uncertainty.

\section{REFERENCES}

[1] M. van Schijndel-de Nooij, B. Krosse, T. van den Broek, S. Maas, E. van Nunen, H. Zwijnenberg, A. Schieben, H. Mosebach, N. Ford,
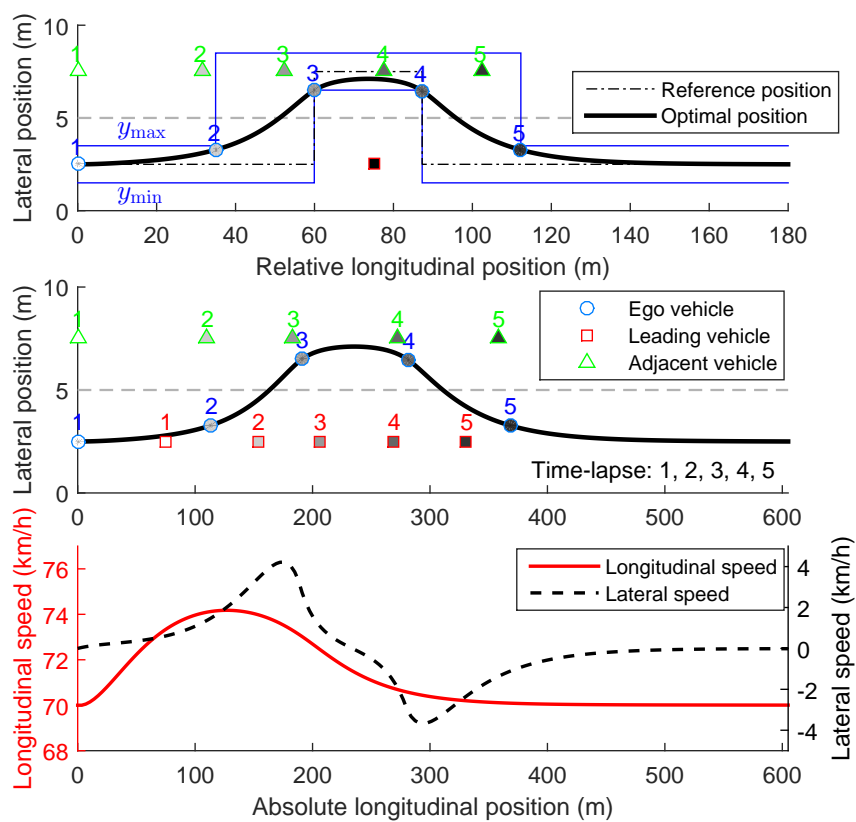

Fig. 5. Overtaking scenario in the proximity of a vehicle driving on an adjacent lane with speed of $70 \mathrm{~km} / \mathrm{h}$. The ego vehicle accelerates to about $74 \mathrm{~km} / \mathrm{h}$ in order to align with the leading vehicle on the adjacent lane, before activating the ramp barrier of the adjacent vehicle.

M. McDonald, D. Jeffery, J. Piao, and J. Sanchez, "Definition of necessary vehicle and infrastructure systems for automated driving," European Commission, Tech. Rep. 2010/0064, 2011.

[2] J. Pohl, W. Birk, and L. Westervall, "A driver-distraction-based lanekeeping assistance system," J. Systems and Control Engineering, vol. 221, pp. 541-552, 2007

[3] Y. Kuwata, G. Fiore, J. Teo, E. Frazzoli, and J. How, "Motion planning for urban driving using RRT," in IEEE/RSJ International Conference on Intelligent Robots and Systems, 2008, pp. 1681-1686.

[4] J. Ziegler, M. Werling, and J. Schröder, "Navigating car-like robots in unstructured environments using an obstacle sensitive cost function," in IEEE Intelligent Vehicles Symposium, 2008, pp. 787-791.

[5] Y. Gao, A. Gray, J. V. Frasch, T. Lin, H. E. Tseng, J. K. Hedrick, and F. Borrelli, "Spatial predictive control for agile semi-autonomous ground vehicles," in 11th International Symposium on Advanced Vehicle Control, 2012.

[6] P. Falcone, H. E. Tseng, F. Borrelli, J. Asgari, and D. Hrovat, "MPC-based yaw and lateral stabilisation via active front steering and braking," Vehicle System Dynamics: International Journal of Vehicle Mechanics and Mobility, vol. 46, pp. 611-628, 2008.

[7] D. Mayne, J. Rawlings, C. Rao, and P. Scokaert, "Constrained model predictive control : Stability and optimality," Automatica, vol. 36, no. 6, pp. 789-814, 2000.

[8] J. Nilsson, M. Ali, P. Falcone, and J. Sjöberg, "Predictive manoeuvre generation for automated driving," in Intelligent Transportation Systems (ITSC), The Hague, The Netherlands, 2013.

[9] J. Nilsson, Y. Gao, A. Carvalho, and F. Borrelli, "Manoeuvre generation and control for automated highway driving," in IFAC World Congress, Cape Town, South Africa, 2014.

[10] S. Boyd and L. Vandenberghe, Convex Optimization. Cambridge University Press, 2004.

[11] I. CVX Research, "CVX: Matlab software for disciplined convex programming, version 2.0 beta," http://cvxr.com/cvx, 2012.

[12] M. Grant and S. Boyd, "Graph implementations for nonsmooth convex programs," in Recent Advances in Learning and Control, ser. Lecture Notes in Control and Information Sciences, V. Blondel, S. Boyd, and H. Kimura, Eds. Springer-Verlag Limited, 2008, pp. 95-110, http://stanford.edu/ boyd/graph_dcp.html.

[13] A. Domahidi, E. Chu, and S. Boyd, "ECOS: An SOCP solver for embedded systems," in European Control Conference (ECC), 2013 pp. 3071-3076. 